\title{
ON THE NON-EXISTENCE OF SOME INTERPOLATORY POLYNOMIALS
}

\author{
C.H. ANDERSON and J. PRASAD \\ Department of Mathematics \\ California State University \\ Los Angeles, California 90032 U.S.A.
}

(Received May 20, 1985)

ABSTRACT. Here we prove that if $x_{k}, k=1,2, \ldots, n+2$ are the zeros of $\left(1-x^{2}\right) T_{n}(x)$ where $T_{n}(x)$ is the Tchebycheff polynomial of first kind of degree $n, \alpha_{j}, \beta_{j}$, $j=1,2, \ldots, n+2$ and $\gamma_{j}, j=2,3, \ldots, n+1$ are any real numbers there does not exist a unique polynomial $Q_{3 n+3}(x)$ of degree $\leq 3 n+3$ satisfying the conditions: $Q_{3 n+3}\left(x_{j}\right)=\alpha_{j}, Q_{3 n+3}^{\prime}\left(x_{j}\right)=\beta_{j}, j=1,2, \ldots, n+2$ and $Q_{3 n+3}^{\prime \prime \prime}\left(x_{j}\right)=\gamma_{j}, j=2,3, \ldots$, $n+1$. Similar result is also obtained by choosing the roots of $\left(1-x^{2}\right) P_{n}(x)$ as the nodes of interpolation where $P_{n}(x)$ is the Legendre polynomial of degree $n$.

KEY WORDS AND PHRASES. Roots, interpolatory polynomials, non-existence, nodes. 1980 AMS SUBJECT CLASSIFICATION CODE. 41 A25.

1. INTRODUCTION.

In [1] R.B. Saxena considered an interesting problem of $(0,1,3)$ interpolation by taking the roots of $\left(1-x^{2}\right) P_{n-2}(x)$, where $P_{n-2}(x)$ is the Legendre polynomial of degree $n-2$, as the nodes of interpolation. By $(0,1,3)$ interpolation, Saxena meant that for the collections $\left\{\alpha_{j}\right\}_{1}^{n},\left\{\beta_{j}\right\}_{2}^{n-1}$, and $\left\{\gamma_{j}\right\}_{1}^{n}$ of real numbers and the zeros $x_{j}$ of $\left(1-x^{2}\right) P_{n-2}(x)$ arranged so that

$$
-1=x_{n}<x_{n-1}<\ldots<x_{2}<x_{1}=1
$$

a polynomial $R_{n}(x)$ of degree $\leq 3 n-3$ can be constructed so that

$$
\begin{aligned}
& R_{n}\left(x_{j}\right)=\alpha_{j} ; j=1,2, \ldots, n, \\
& R_{n}^{\prime}\left(x_{j}\right)=\beta_{j} ; j=2,3, \ldots, n-1,
\end{aligned}
$$

and

$$
R_{n}^{\prime \prime \prime}\left(x_{j}\right)=\gamma_{j} ; j=1,2, \ldots, n \text {. }
$$

Saxena proved that such a polynomial exists uniquely if $n$ is even and for $n$ odd there does not exist a unique polynomial $R_{n}(x)$ satisfying the above conditions. Later Varma [2] obtained the following result in this direction:

THEOREM 1 (VARMA). Given a positive integer $n$ and real numbers $\alpha_{k}(k=1,2, \ldots$, $n+2), \beta_{k}, \gamma_{k}(k=2,3, \ldots, n+1)$ there is, in general no polynomial $F_{3 n+1}(x)$ of degree $\leq 3 n+1$ such that $F_{3 n+1}\left(x_{k}\right)=\alpha_{k} ; k=1,2, \ldots, n+2, F_{3 n+1}^{\prime}\left(x_{k}\right)=\beta_{k}$; 
$k=2,3, \ldots, n+1$ and $F_{3 n+1}^{\prime \prime \prime}\left(x_{k}\right)=\gamma_{k} ; k=2,3, \ldots, n+1$ provided $x_{k}^{\prime}$ s are the zeros of $\left(1-x^{2}\right) T_{n}(x)$ where $T_{n}(x)$ is Tchebycheff polynomial of first kind and if there exists such a polynomial then there is an infinity of them.

2. MAIN RESULTS.

In connection with the above results we shall prove the following.

THEOREM 2. For any positive integer $n$, with $1=\xi_{1}>\xi_{2}>\ldots>\xi_{n+1}>\xi_{n+2}$ $=-1$ the zeros of $\left(1-x^{2}\right) P_{n}(x)$ where $P_{n}(x)$ is the Legendre polynomial of degree $n$, there is in general no polynomial $R_{3 n+1}(x)$ of degree $\leq 3 n+1$ such that, for arbitrary real numbers $\left\{\alpha_{j}\right\}_{1}^{n+2},\left\{\beta_{j}\right\}_{2}^{n+1}$ and $\left\{\gamma_{j}\right\}_{2}^{n+1}$ the conditions:

$$
\begin{aligned}
& R_{3 n+1}\left(\xi_{j}\right)=\alpha_{j} ; j=1,2, \ldots, n+1, n+2, \\
& R_{3 n+1}^{\prime}\left(\xi_{j}\right)=\beta_{j} ; j=2,3, \ldots, n+1
\end{aligned}
$$

and

$$
R_{3 n+1}^{\prime \prime \prime}\left(\xi_{j}\right)=\gamma_{j} ; j=2,3, \ldots, n+1
$$

are satisfied. If there does exist such a polynomial then there are infinitely many of them.

We'also prove the following result for Tchebycheff nodes:

THEOREM 3. For any positive integer $n$, with $1=x_{1}>x_{2}>\ldots .>x_{n}>x_{n+1}$ $>x_{n+2}=-1$ the zeros of $\omega_{n}(x)=\left(1-x^{2}\right) T_{n}(x)$, there is in general no polynomial $Q_{3 n+3}(x)$ of degree $\leq 3 n+3$ such that for arbitrary real numbers $\left\{\alpha_{j}\right\}_{1}^{n+2},\left\{\beta_{j}\right\}_{1}^{n+2}$ and $\left\{\gamma_{j}\right\}_{2}^{n+1}$ the conditions:

$$
\begin{aligned}
& Q_{3 n+3}\left(x_{j}\right)=\alpha_{j} ; j=1,2, \ldots, n+1, n+2, \\
& Q_{3 n+3}^{\prime}\left(x_{j}\right)=B_{j} ; j=1,2, \ldots, n+1, n+2
\end{aligned}
$$

and

$$
Q_{3 n+3}^{\prime \prime \prime}\left(x_{j}\right)=\gamma_{j} ; j=2,3, \ldots, n+1
$$

are satisfied. If there does exist such a polynomial then there are infinitely many of them.

REMARK 1. The comparison of our Theorem 2 with the above mentioned result of Saxena shows that if we do not prescribe the third derivative at \pm 1 then there does not exist a unique polynomial regardless whether $n$ is even or odd. In an earlier work [3] we have shown that along with the conditions (2.1), (2.2) and (2.3) if we also prescribe the first derivative at \pm 1 a unique polynomial of degree $\leq 3 n+3$ still does not exist. It is also evident from Theorem 3 that even if we prescribe the first derivative at \pm 1 a unique polynomial of degree $\leq 3 n+3$ does not exist although the nodes of interpolation are different from that of [3].

REMARK 2. We shall give here the proof of Theorem 3 only. The proof of Theorem 2 can be obtained along the same lines.

PROOF OF THEOREM 3. We will show that if all of

$$
\begin{aligned}
& \alpha_{j}=0 ; j=1,2, \ldots, n+1, n+2, \\
& \beta_{j}=0 ; j=1,2, \ldots, n+1, n+2, \\
& \dot{\gamma}_{j}=0 ; j=2,3, \ldots, n+1
\end{aligned}
$$


then there exists a polynomial $Q_{3 n+3}(x)$ of degree $\leq 3 n+3$ which is not identically zero, but satisfies $(2.4),(2.5)$ and (2.6). The desired result then follows immediately from the theory of linear equations. From the definition of $\omega_{n}(x)$ and conditions $(2.4),(2.5)$ and $(2.6)$, together with the requirements (2.7), it is clear that the desired polynomial must be of the form

$$
Q_{3 n+3}(x)=\left(1-x^{2}\right)^{2} T_{n}^{2}(x) k_{n-1}(x)
$$

where $h_{n-1}(x)$ is an unknown polynomial of degree $\leq n-1$. Since we have also required $Q_{3 n+3}^{\prime \prime \prime}\left(x_{j}\right)=0$; for $\mathrm{J}=2,3, \ldots, n+1$, simple calculation provides

$$
\left(1-\mathrm{x}^{2}\right) k_{\mathrm{n}-1}^{\prime}(\mathrm{x})-3 \mathrm{x} \kappa_{\mathrm{n}-1}(\mathrm{x})=\mathrm{cT}_{\mathrm{n}}(\mathrm{x})
$$

for unknown real constant c. Letting $x=\cos \theta$ and

$$
r_{n-1}(x)=\sum_{k=0}^{n-1} a_{k} \cos k \theta
$$

we obtain

$$
\left(1-x^{2}\right) r_{n-1}^{\prime}(x)=\sum_{k=1}^{n-1} a_{k} k \sin k \theta \sin \theta .
$$

Thus (2.9) becomes

$$
c \cos n \theta=\sum_{k=0}^{n-1} a_{k}[k \sin k \theta \sin \theta-3 \cos k \theta \cos \theta] \text {. }
$$

From this, we obtain on simplification

$$
2 c \cos n \theta=\sum_{k=0}^{n-1} a_{k}[(k-3) \cos (k-1) \theta-(k+3) \cos (k+1) \theta],
$$

from which, by collecting the coefficients of $\cos k \theta$, for $k=0,1, \ldots, n$, we may write

$$
\begin{aligned}
& -2 a_{1}-\left(6 a_{0}+a_{2}\right) \cos \theta-4 a_{1} \cos 2 \theta \\
& +\sum_{k=3}^{n-2}\left\{(k-2) a_{k+1}-(k+2) a_{k-1}\right\} \cos k \theta \\
& -(n+1) a_{n-2} \cos (n-1) \theta-(n+2) a_{n-1} \cos n \theta \\
& =2 c \cos n \theta .
\end{aligned}
$$

This, in turn, leads to the following system of equations

$$
\begin{aligned}
& -2 a_{1}=0 \\
& -\left(6 a_{0}+a_{2}\right)=0, \\
& -4 a_{1}=0, \\
& (k-2) a_{k+1}-(k+2) a_{k-1}=0 ; k=3,4, \ldots, n-2, \\
& -(n+1) a_{n-2}=0, \\
& -(n+2) a_{n-1}=2 c .
\end{aligned}
$$

If $\mathrm{n}$ is even, then

$$
a_{0}=a_{2}=a_{4}=\ldots \ldots=a_{n-2}=0 ; a_{1}=0
$$


but

$$
a_{n-1-2 j}=\frac{-2 c}{n-2} \prod_{k=0}^{j}\left(\frac{n-2-2 k}{n+2-2 k}\right) ; \text { for } j=0,1, \ldots,(n-4) / 2
$$

is not necessarily zero.

If $\mathrm{n}$ is odd, then

$$
a_{1}=a_{3}=a_{5}=\ldots .=a_{n-2}=0 \text {, }
$$

while

$$
a_{2 j}=\frac{-2 c}{n-2} \prod_{k=j}^{(n-1) / 2} \frac{2 k-1}{2 k+3} ; j=1,2, \ldots, \frac{(n-1)}{2}
$$

with the special case

$$
a_{0}=-a_{2} / 6
$$

which are not necessarily zero. Hence regardless whether $\mathrm{n}$ is even or odd, in general, there does not exist a unique polynomial $Q_{3 n+3}(x)$ of degree $\leq 3 n+3$ satisfying (2.4), (2.5) and (2.6) and there are infinitely many if they exist.

This completes the proof of Theorem 3. For a complete history on lacunary interpolation we refer to a paper by J. Balázs [4].

\section{REFERENCES}

1. SAXENA, R.B., On some interpolatory properties of Legendre polynomials III, Academic Bulgare Des Sciences Bul1. De L'Institut De Math. 8 (1964), 63-94.

2. VARMA, A.K., Non-existence of interpolatory polynomials, Publ. Math... (Debrecan) 15 (1968), 75-77.

3. PRASAD, J. AND ANDERSON, C.H., Some remarks on non-existence of interpolation polynomials, Math. Notae Vol. 24 (1981-1982), 67-72.

4. BALÁZS, J., Sûlyozott $(0,2)$ interpolácio Ultraszférikus polinomok gyökeim, Magyar Tud. Akad. Mat. Fiz. Oszt. Köz1. 11 (3) (1961), 305-338. 


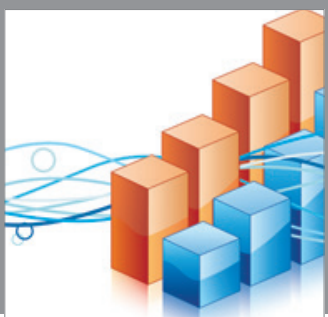

Advances in

Operations Research

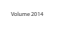

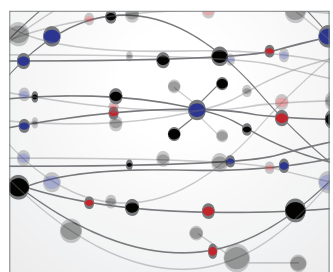

\section{The Scientific} World Journal
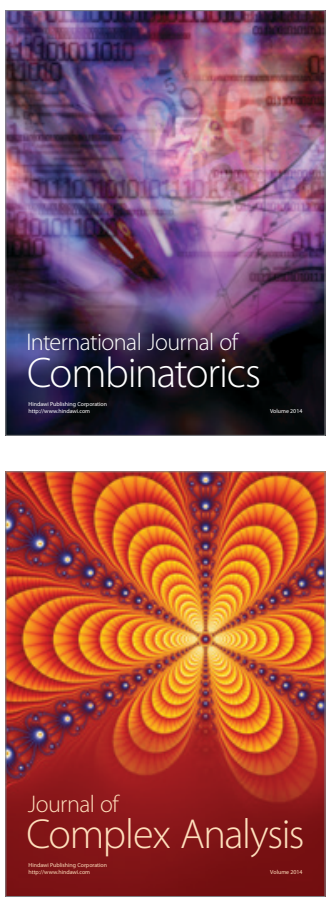

International Journal of

Mathematics and

Mathematical

Sciences
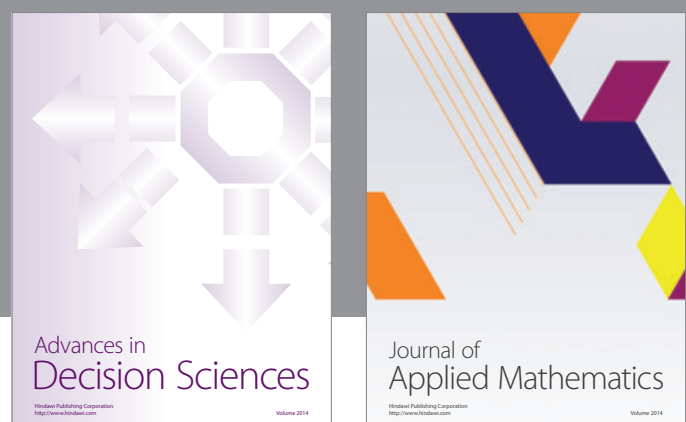

Journal of

Applied Mathematics
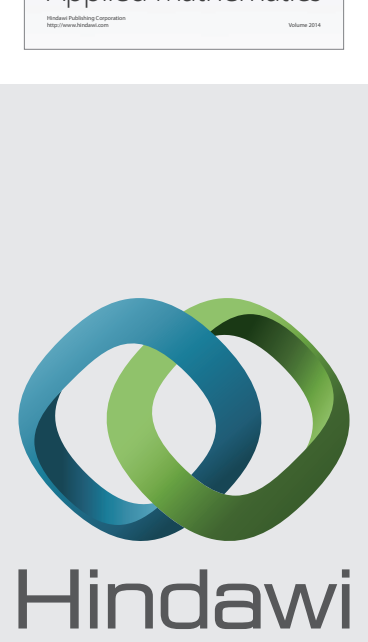

Submit your manuscripts at http://www.hindawi.com
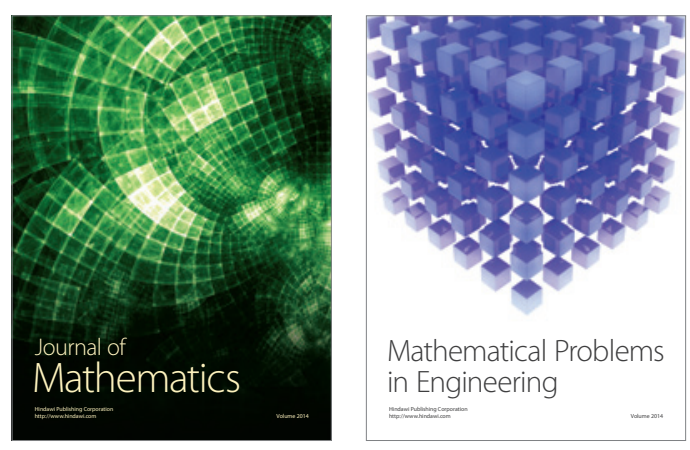

Mathematical Problems in Engineering
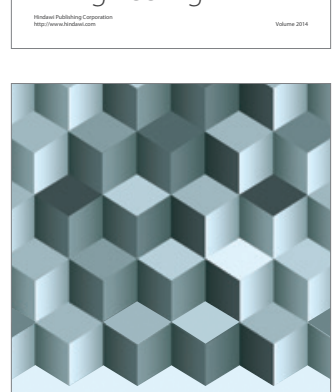

Journal of

Function Spaces
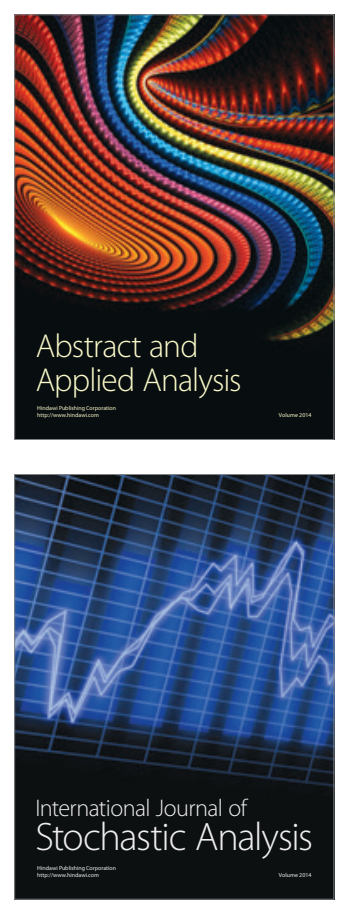

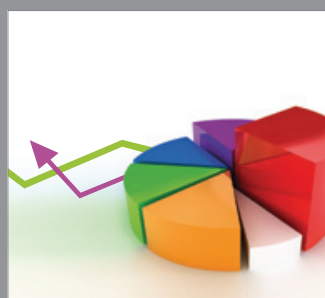

ournal of

Probability and Statistics

Promensencen
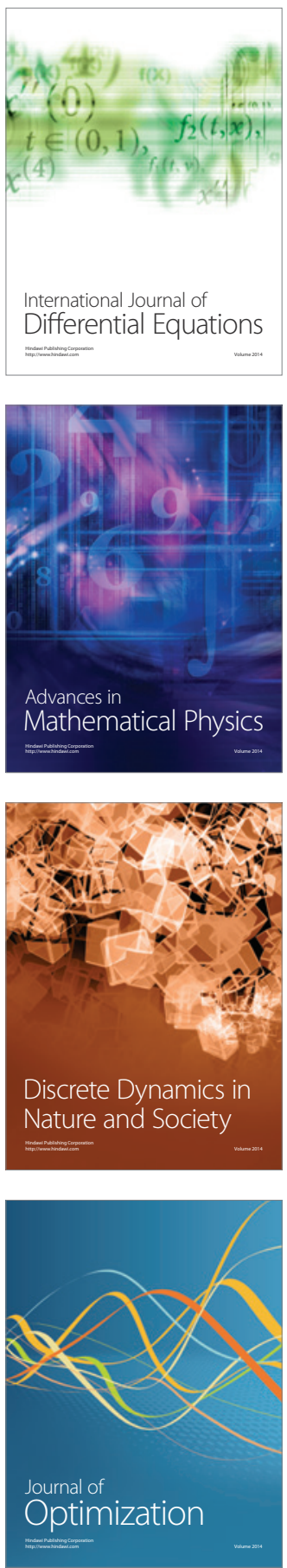